\title{
High-resolution mapping of genes involved in plant stage-specific partial resistance of barley to leaf rust
}

\author{
F. K. S. Yeo • R. Bouchon • R. Kuijken • A. Loriaux • \\ C. Boyd • R. E. Niks • T. C. Marcel
}

Received: 21 November 2015 / Accepted: 20 January 2017 /Published online: 16 March 2017

(C) The Author(s) 2017. This article is published with open access at Springerlink.com

\begin{abstract}
Partial resistance quantitative trait loci (QTLs) Rphq11 and rphq16 against Puccinia hordei isolate 1.2.1 were previously mapped in seedlings of the mapping populations Steptoe/Morex and Oregon Wolfe Barleys, respectively. In this study, QTL mapping was performed at adult plant stage for the two
\end{abstract}

Electronic supplementary material The online version of this article (doi:10.1007/s11032-017-0624-x) contains supplementary material, which is available to authorized users.

F. K. S. Yeo · R. Bouchon · R. Kuijken · A. Loriaux •

R. E. Niks $(\square) \cdot$ T. C. Marcel

Plant Breeding, Wageningen University and Research, Droevendaalsesteeg 1, 6708PB, 6700 AJ Wageningen, the Netherlands

e-mail: rients.niks@wur.nl

\section{F. K. S. Yeo}

Department of Plant Science and Environmental Ecology, Faculty of Resource Science and Technology, University Malaysia

Sarawak, 94300 Kota Samarahan, Sarawak, Malaysia

C. Boyd

Department of Crop and Soil Sciences, Washington State

University, Pullman, WA, 99164-4660, USA

T. C. Marcel

UMR BIOGER, INRA, AgroParisTech, Université Paris-Saclay, 78850 Thiverval-Grignon, France

Present Address:

R. Kuijken

Greenhouse Horticulture, Wageningen University and Research, 6700 AP, Wageningen, the Netherlands mapping populations challenged with the same rust isolate. The results suggest that Rphq11 and rphq16 are effective only at seedling stage, and not at adult plant stage. The cloning of several genes responsible for partial resistance of barley to $P$. hordei will allow elucidation of the molecular basis of this type of plant defence. A map-based cloning approach requires to fine-map the QTL in a narrow genetic window. In this study, Rphq11 and rphq16 were fine-mapped using an approach aiming at speeding up the development of plant material and simplifying its evaluation. The plant materials for fine-mapping were identified from early plant materials developed to produce QTL-NILs. The material was first selected to carry the targeted QTL in heterozygous condition and susceptibility alleles at other resistance QTLs in homozygous condition. This strategy took four to five generations to obtain fixed QTL recombinants (i.e., homozygous resistant at the Rphq11 or rphq16 QTL alleles, homozygous susceptible at the non-targeted QTL alleles). In less than 2 years, Rphq11 was fine-mapped into a $0.2-\mathrm{cM}$ genetic interval and a 1.4-cM genetic interval for rphq16. The strongest candidate gene for Rphq11 is a phospholipid hydroperoxide glutathione peroxidase. Thus far, no candidate gene was identified for rphq16.

Keywords High resolution mapping - Quantitative trait locus (QTL) · Puccinia · Barley · Resistance 


\section{Introduction}

Partial resistance of barley against barley leaf rust (Puccinia hordei) results in a reduced epidemic, despite a compatible infection type (Parlevliet 1979). The reduced rate of epidemic progress is due to a lower infection frequency, lower sporulation rate and longer latency period of the pathogen on barley accessions with high levels of partial resistance (Parlevliet 1979). Partial resistance is a prehaustorial resistance, where failed attempts to form haustoria are associated with cell wall reinforcements, called papillae (Niks 1986; O'Connell and Panstruga 2006). The failure of a proportion of the haustorium formation reduces the capacity for nutrient extraction from the plant and for delivery of pathogenicity-promoting effectors into the plant cells (Niks 1986; Catanzariti et al. 2007; de Jonge et al. 2011). This prehaustorial mechanism of resistance is also implicated in non-host resistance of barley to heterologous rust fungi like Puccinia recondita and P. triticina (Niks 1983, 1989).

Partial resistance of barley to $P$. hordei is polygenically inherited and is supposed to act on a minor-gene-forminor-gene model (González et al. 2012; Marcel et al. 2008; Niks et al. 2000; Parlevliet and Zadoks 1977; Qi et al. 1999; Niks et al. 2015). There is an abundance of quantitative trait loci (QTLs) for partial resistance against barley leaf rust. To date, at least 20 partial resistance QTLs against barley leaf rust have been mapped in different biparental mapping populations. In each barley mapping population, a different set of QTLs was identified, with few QTLs shared among the populations. The explained phenotypic variation per QTL ranges from around 50 to 50\% (Jafary et al. 2008; Marcel et al. 2008; Marcel et al. 2007b; Niks et al. 2000; Qi et al. 1999; Qi et al. 1998).

QTL mapping studies indicated that the resistance QTLs can be effective across different stages of plant development or only at specific stages (Qi et al. 1998), which was confirmed on QTLs that were introgressed into near isogenic lines (NILs) (Wang et al. 2010). Plant growth stage-dependent effects of resistance QTLs have also been observed in other plant pathosystems (Aghnoum et al. 2010; Dedryver et al. 2009; Shankar et al. 2008; Steffenson et al. 1996). Consequently, different sets of QTLs will protect barley plants against leaf rust at different growth stages. It is important to know the effect of QTLs at different growth stages before designing breeding strategies or to engage in a positional cloning procedure.

The cloning of several genes responsible for partial resistance of barley to $P$. horde $i$ will allow elucidation of the molecular basis of this type of plant defence (Marcel et al. 2007a). Fine mapping and positional cloning require the evaluation of very large numbers of plants in a controlled environment and a similar physiological condition, which is much easier to achieve at seedling stage. To date, no QTL for resistance to rust fungi or powdery mildew has been cloned in barley. However, three largeeffect resistance QTLs have been cloned in rice: two against Magnaporthe oryzae (Fukuoka et al. 2009; Hayashi et al. 2010) and one wide-spectrum QTL against Rhizoctonia solani and M. oryzae (Manosalva et al. 2009). There are three cloned QTLs in wheat; one against Puccinia striiformis (Fu et al. 2009) and two wide-spectrum QTLs against the three rust species $P$. triticina, $P$. striiformis and $P$. graminis and against Blumeria graminis (Krattinger et al. 2009; Moore et al. 2015). All the genes cloned so far belong to different gene families and are involved in different molecular functions, suggesting a wide diversity of mechanisms underlying partial resistance.

A map-based cloning approach requires to fine-map the QTL in a genetic window sufficiently narrow to make physical mapping feasible. This approach requires the effect of the QTL to be sufficiently clear to infer the QTL genotype from its phenotype; the phenotypic variation explained by the QTL should be more than $10 \%$ according to Kou and Wang (2012). Once identified in a segregating mapping population, the commonly followed strategy to fine-map QTLs requires the introgression of the QTL in a NIL. In non-isogenic plant materials, other QTLs may be segregating in the genetic background blurring the determination of the phenotypic effect of the QTL of interest. The NIL carrying the targeted QTL is crossed with its recurrent parent to "Mendelise" the QTL in the resulting progeny. Then, a selection of plants recombining at the QTL-containing chromosome region (i.e., sub-NILs) and the evaluation of their phenotype allow to pinpoint the targeted QTL into a refined genetic position (Han et al. 1999; Marcel et al. 2007a; Xue et al. 2010; Zhou et al. 2010). Fine mapping using this strategy is effective but very laborious and time consuming in generating the plant materials, marker development and genotyping.

$R p h q 11$ (resistance is dominant) and rphq16 (resistance is recessive) are partial resistance QTLs against $P$. hordei isolate 1.2.1 that were mapped in seedlings of the mapping populations Steptoe/Morex (S/M) and Oregon Wolfe Barleys (OWB; Dom/Rec), respectively (Marcel et al. 2007b). They are effective at seedling stage in their 
respective mapping population, each explaining approximately $30 \%$ of the phenotypic variance. Rphq11 was mapped at seedling stage near the middle of chromosome $2 \mathrm{HL}$, and the resistance allele was contributed by Steptoe. It was also detected by Chen et al. (2010) as an expression QTL (eQTL) co-locating with the phenotypic QTL (pQTL) in the same mapping population. Six candidate genes were suggested by those authors that may explain Rphq11. rphq16 was mapped at seedling stage near the telomeric region of chromosome 5HL, and the resistance allele was contributed by Dom.

The first objective of this study was to test whether Rphq11 and rphq16 are also effective at adult plant stage. The second objective was to fine-map Rphq11 and $r p h q 16$ using an approach aimed at speeding up the development of plant material and simplifying its evaluation with the final aim of cloning them. Rphq11 and rphq16 qualify for map-based cloning to study partial resistance because of sufficient size of phenotypic effect at seedling stage.

\section{Materials and Methods}

Inoculum

All the disease tests in this study were done with P. hordei isolate 1.2.1 (Ph.1.2.1), the same isolate as used by Marcel et al. (2007b). This isolate is a monospore purification of the isolate 1.2 collected in the Netherlands in 1971 (Parlevliet 1976).

QTL mapping on adult plants

The doubled haploid (DH) mapping populations S/M (Kleinhofs et al. 1993) and OWB (Costa et al. 2001) were used to map QTLs for non-hypersensitive quantitative resistance at adult plant developmental stage, heading stage Z51-Z55 on Zadoks' growth scale (Zadoks et al. 1974). The prehaustorial, nonhypersensitive resistance conferred by Rphq11 and rphq16 was confirmed by microscopic observations (data not shown). The S/M population comprises 150 DH lines, and the OWB population comprises $94 \mathrm{DH}$ lines. For QTL mapping, all marker positions were extracted from the "Barley, Integrated, Marcel 2009" linkage map [linkage map and segregating marker data are available at http://wheat.pw.usda.gov/GG2/index. shtml; (Aghnoum et al. 2010)]. Data comprise 3561 segregating markers in S/M and 882 in OWB. Skeletal maps were generated for $\mathrm{S} / \mathrm{M}$ and $\mathrm{OWB}$ by selecting markers homogeneously distributed over the integrated map, spaced at approximately $1-5-\mathrm{cM}$ intervals.

The disease phenotyping of the entire populations was repeated three times - at different periods of the year. In the first and second repetitions, we evaluated three individual plants (biological replicates) per DH line. The third repetition was performed at a different glasshouse facility regulated with the same climatic conditions, and only one individual plant per DH line was evaluated. The parental lines Steptoe and Morex or Dom and Rec and the reference barley lines L94 and Vada were included in each experiment. Parental and reference lines were sown continuously every 3 days from 1 week before to 1 week after the sowing of the DH lines of the two mapping populations. For each line, three seeds were sown in a pot of $15 \mathrm{~cm}$ diameter and $14 \mathrm{~cm}$ height. For the first and second repetitions, all three plants in a pot were scored. For the third repetition, one out of three plants was selected randomly for scoring. To ensure the uniformity of the developmental stage of the plants at the time of inoculation, plants of a mapping population were divided into three to four subgroups based on their heading date. For each subgroup, plants of parental and reference lines with a similar developmental stage were added.

Plants were inoculated after the flag leaf was unfolded (around heading stage Z52). Per pot, $1 \mathrm{mg}$ of spores diluted 10 times with Lycopodium spores was used as inoculum. Before the inoculation, the pots were lined up two by two. Then, the inoculum was dusted over the plants as uniformly as possible. The inoculated plants were then placed in a humidity chamber overnight $(8 \mathrm{~h})$ at $100 \%$ relative humidity in the dark at $18{ }^{\circ} \mathrm{C}$ to allow the spores to germinate. After incubation, the plants were transferred to a greenhouse compartment where the temperature was set at $20 \pm 3{ }^{\circ} \mathrm{C}$ during daytime and about $16^{\circ} \mathrm{C}$ at night, with $30-70 \%$ relative humidity.

The flag leaf of the three plants in each pot was scored for latency period (LP50A). It was scored daily by counting the mature pustules on a marked area of the flag leaf until all the pustules matured. Immature colonies are seen as small pale green or yellowish flecks on the leaves. Those flecks turn orange at maturation, indicating the beginning of sporulation by the colony (Niks et al. 2015). Latency period estimates the period of time in hours at which $50 \%$ of the total number of pustules are mature. It is among the most informative measures 
of barley partial resistance to leaf rust and is relatively easy to evaluate (Niks et al. 2011; Parlevliet 1979). The relative latency period (RLP50A) was calculated, relative (in \%) to the LP50A on Steptoe for S/M and to the LP50A on Dom for OWB.

ANOVA was performed using GenStat ${ }^{\circledR}$ 14th edition (VSN International Ltd. 2011). QTLs were mapped using MapQTL@6 (van Ooijen 2009). QTL mapping was performed for each repetition independently. A permutation test with 1000 permutations was performed for each repetition to set the LOD threshold to declare a QTL. The confidence interval of a QTL is the estimated LOD-2 support interval.

The data for heading date (HD) and plant height (PH) of $\mathrm{S} / \mathrm{M}$ were downloaded from GrainGenes (http://wheat.pw.usda.gov/ggpages/SxM/phenotypes.html) and described by Hayes et al. (1993). The QTLs for HD and $\mathrm{PH}$ were mapped on the $\mathrm{S} / \mathrm{M}$ skeletal map generated from the barley integrated map [(Barley, Integrated, Marcel 2009; (Aghnoum et al. 2010)].

Dominance of phenotypic expression for the target QTLs

Previous QTL mapping studies at seedling stage revealed in the S/M population two QTLs, Rphq11 and Rphq15, for which the resistance alleles were contributed by Steptoe. In the OWB population, resistance alleles of two QTLs, rphq16 and Rphq17, were contributed by Dom (Marcel et al. 2007b). Steptoe and Dom were individually crossed with the experimental line SusPtrit, and their $F_{1}$ backcrossed to SusPtrit (Atienza et al. 2004). Molecular markers flanking the QTLs were used to select for the susceptibility QTL alleles at Rphq15 and Rphq17 and to select for the resistance QTL alleles at Rphq11 and rphq16. Rphq11 and rphq16 were finally introgressed into SusPtrit by backcrossing over five generations for Rphq11 and six generations for rphq16 to obtain NILs (Supplementary Material, Fig. S1). Details and results obtained with the NILs will be published elsewhere.

Steptoe/SusPtrit $\mathrm{F}_{2}$ plants being heterozygous at $R p h q 11$ and homozygous susceptible at Rphq15 were selected with three molecular markers for Rphq11 (Bmag0125, GBM1062, GBMS244; cf. Supplementary Material Table S1) and three molecular markers for rphq15 (scssr09398, GBM033, MWG966; Supplementary Material Table S3). Three $\mathrm{F}_{2}$ plants were selected from which $97 \mathrm{~F}_{3}$ plants were genotyped with flanking markers and phenotyped to perform a dominance study of Rphq11 (Supplementary Material, Fig. S1).

$\mathrm{Dom} /$ SusPtrit $\mathrm{BC}_{1}$ plants being heterozygous at Rphq16 and homozygous susceptible at Rphq17 were selected with four molecular markers for rphq16 (ABG390, ABG391, GMS002, scssr09041; cf. Supplementary Material Table S2) and two microsatellite markers for rphq17 (Bmac0067, Bmag0136; Supplementary Material Table S3). Two $\mathrm{BC}_{1}$ plants were selected from which $52 \mathrm{BC}_{1} \mathrm{~S}_{1}$ plants were genotyped with flanking markers and phenotyped to perform a dominance study of $r p h q 16$ (Supplementary Material, Fig. S1).

The first leaves of the $97 \mathrm{~F}_{3}$ seedlings for Rphq11 and $52 \mathrm{BC}_{1} \mathrm{~S}_{1}$ seedlings for rphq16 were inoculated with Ph.1.2.1 following the method of Qi et al. (1998). Their latency period was scored (LP50S) and the relative latency period (RLP50S) calculated, relative to SusPtrit (in \%). The plants were grouped according to their QTL allele, homozygous Steptoe or Dom (AA), and heterozygous (AB) and homozygous SusPtrit (BB), to estimate the effect and the dominance or recessiveness of Rphq11 and rphq16. Plants that had a recombination between the QTL flanking markers were excluded from the analysis. The data were analysed with unbalanced one-way ANOVA using GenStat ${ }^{\circledR}$ 14th edition (VSN International Ltd. 2011).

Fine mapping Rphq11 and rphq16

Among the $97 \mathrm{~F}_{3}$ plants for Rphq11 and among the 52 $\mathrm{BC}_{1} \mathrm{~S}_{1}$ plants for rphq16, there were 12 and 18 recombinant plants, respectively. These plants were grown to set seeds which were then used to identify plants with homozygous recombination. These plants were then homozygous recombinants at the Rphq11 and rphq16 QTL regions, homozygous susceptible at the Rphq15 and Rphq17 QTL regions, but their genomic background was still segregating. For simplicity, these plants will be called "fixed QTL recombinants" from this point onwards. These fixed QTL recombinants were used to refine the positions of Rphq11 and rphq16 (data not shown). Based on the refined positions, new flanking markers were selected for Rphq11 (GBS0512 and GBMS244) and for rphq16 (scsnp03275 and GMS002) (Supplementary Material Tables S1 and S2).

In order to further fine-map Rphq11 and rphq16, the $\mathrm{F}_{3}$ plants heterozygous for Rphq11 and $\mathrm{BC}_{2}$ plants heterozygous for $r p h q 16$ were selfed to produce a large number of seeds. New recombinants for Rphq11 and 
rphq16 were identified by screening the $\mathrm{F}_{4}$ and $\mathrm{BC}_{2} \mathrm{~S}_{1}$ plants with the new flanking markers for Rphq11 (GBS0512 and GBMS244) and rphq16 (scsnp03275 and GMS002). The same markers were used to identify fixed QTL recombinants for both QTLs in the subsequent generation. The fixed QTL recombinants were then genotyped with all available molecular markers located in the QTL regions to generate high-resolution genetic maps around Rphq11 and rphq16.

The fixed QTL recombinants were subjected to four rounds of disease tests for Rphq11 and three for rphq16 (Supplementary Material, Fig. S2). At each round, a different subset of the fixed QTL recombinants was strategically selected based on previous results of disease tests in order to progressively refine the map position of the QTL. This strategy allowed more individuals to be tested per fixed QTL recombinant at each round (from 5 to 10 individuals), increasing the confidence in the phenotype. SusPtrit was included in all disease tests as susceptible reference. The order of the fixed QTL recombinants was randomised within each experimental round, but individuals from the same fixed QTL recombinant were sown next to each other.

Disease tests were performed at seedling stage following the method of Qi et al. (1998). The LP50S was measured. The relative latency period on seedlings (RLP50S) was calculated by setting SusPtrit at 100 . Data from different rounds of disease test were analysed together under a linear mixed model with GenStat ${ }^{\circledR}$ 14th edition (VSN International Ltd. 2011). The significance of difference in mean RLP50S between fixed QTL recombinants and SusPtrit was determined based on the least significant difference (LSD, $P<0.05$ ).

To recover rare recombinant plants, genomic DNA of the plant materials for recombinant screening was extracted following the method of Wang et al. (1993), adjusted for a 96-well format. To select fixed QTL recombinants, the sbeadex ${ }^{\circledR}$ maxi plant kit (LGC Genomics) was used to isolate DNA of recombinant plants.

\section{Marker saturation of Rphq11 and rphq16 intervals}

Two approaches were followed to develop 20 molecular markers in the approximately 13-cM interval of Rphq11 and to develop 27 molecular markers in the approximately 25-cM interval of rphq16. All the markers developed were polymorphic in SusPtrit/Steptoe and SusPtrit/Dom, respectively.
Approach I Molecular markers that mapped within the intervals of Rphq11 and rphq16 on the integrated map (GrainGenes: Barley, Integrated, Marcel 2009) were targeted for generating new PCR-based markers segregating in our material. Sequence information of targeted restriction fragment length polymorphism (RFLP) markers and transcript-derived markers (TDMs) was used to design specific primer pairs. For RFLPs, sequences were downloaded from the GrainGenes database (http://wheat.pw.usda.gov/GG2/index.shtml). For TDMs, unigene sequences were downloaded from the Barley SNP Database (http://germinate.scri.ac.uk/barley_ snpdb/dbStats_contig.html) (Potokina et al. 2008). For sequence-tagged site (STS) markers, the primer sequences were obtained directly from the GrainGenes database. The primer sequences of the simple sequence repeat (SSR) markers were obtained from literature (Varshney et al. 2007). Sequence for the Diversity Arrays Technology (DArT) marker ctg15632 and primers for the Cleaved Amplified Polymorphic Sequence (CAPS) marker Uni19962 have been reported elsewhere (Boyd and Horsley 2007).

Approach II Conserved microsynteny between barley, rice, and Brachypodium distachyon was also used to generate new markers closely linked to Rphq11 and rphq16. The sequences of EST-based markers mapped in the vicinity of Rphq11 and rphq16 were used for BLAST searches against rice and $B$. distachyon genome databases; i.e., the Rice Genome Annotation Project BLAST search (http://rice.plantbiology.msu.edu/analyses_ search_blast.shtml) and the B. distachyon BLAST portal (http://plants.ensembl.org/Brachypodium_distachyon). Rice and $B$. distachyon gene sequences within the identified synteny blocks were in turn used for BLAST against the barley EST tentative consensus (TC) sequences from the barley TIGR Gene Indices database (http://compbio.dfci.harvard.edu/tgi/). Only barley TC sequences with a BLAST hit having an E-value $\leq 10^{-15}$ were further considered for primer design and marker development. To maximise the chance of developing markers that map in the target regions of the barley genome, only barley TC sequences having an homologous gene in the syntenic regions of both rice and $B$. distachyon were further considered for primer design and marker development.

Primers were designed using the Lasergene software (DNASTAR® 8 Inc., Madison, WI, USA). For each primer pair, a gradient PCR was performed to determine 
the optimal annealing temperature. Sequencecharacterized amplified region (SCAR) markers were obtained by finding length polymorphism or allelespecific amplification directly after PCR on parental lines. For primers that amplified fragments of the same size in parental lines, CAPS markers were developed. The PCR products were sent for sequencing (BaseClear, Leiden, the Netherlands). SNPs were identified from the sequence obtained, using the Lasergene software. The dCAPS finder program [http://helix.wustl.edu/ dcaps/dcaps.html; (Neff et al. 2002)] was then used to find discriminating restriction enzymes.

Markers developed based on TDMs, synteny, and eQTL candidate genes were named with the prefix WBE for Wageningen Barley ESTs.

\section{Results}

Mapping QTL for partial resistance at adult stage in S/M and OWB populations

A significant repetition $\times$ genotype effect was observed for the adult plant disease tests of both $\mathrm{S} / \mathrm{M}$ and OWB mapping populations. Consequently, QTL mapping was performed for each repetition independently. In both populations and in each repetition, RLP50A showed a continuous distribution of phenotypes with transgressive segregation (Supplementary Material, Fig. S3). On Steptoe and Rec, RLP50A was always higher than on Morex and Dom, respectively, except in the first repetition of the OWB population where Rec and Dom had nearly the same RLP50A. A permutation test suggested a LOD threshold of 3 for each repetition. A QTL was declared only when its LOD profile surpassed this threshold in at least two repetitions of the same population.

Two partial resistance QTLs were mapped in S/M, on chromosomes $1 \mathrm{H}$ and $3 \mathrm{H}$ (Table 1). These two QTLs were mapped in regions where no partial resistance QTL was reported before (Supplementary Material, Fig. S4). They are designated as $R p h q 22$ and $R p h q 23$, respectively. Rphq22 was detected as significant (LOD $>3)$ QTL in all three repetitions of the disease test. Rphq23 was mapped in two repetitions of the disease test while its LOD score in the third repetition was just below the threshold. Rphq22 explained approximately $26 \%$ of the phenotypic variation and $R p h q 23$ explained around $22 \%$. For both QTLs, the resistance allele was donated by Steptoe. No QTL resistance allele was found to be contributed by Morex despite the observed transgressive segregation in the mapping population. This most probably indicates the presence of QTLs with effects too small to be detected in this experiment. The QTLs for heading date (HD) and plant height $(\mathrm{PH})$ segregating in $\mathrm{S} / \mathrm{M}$ were also positioned on the integrated map. Rphq22 and Rphq23 collocate neither with the HD nor the PH QTLs.

For OWB, the Pearson coefficient correlation of RLP50A between repetitions was very low (data not presented). There was no QTL identified in at least two repetitions of the disease test. In each repetition, a different unique QTL was identified, on chromosomes $2 \mathrm{H}, 5 \mathrm{H}$ and $7 \mathrm{H}$, respectively.

In none of the three repetitions of disease tests for $\mathrm{S} / \mathrm{M}$ and OWB, we detected our target QTLs Rphq11 and rphq16. Therefore, it is likely that these two QTLs are effective only at seedling stage (Marcel et al. 2007b), and not at adult plant stage.

Markers developed for Rphq11 and rphq16

Twenty markers were developed that supposedly mapped on chromosome 2HL in the region of Rphq11 flanked by the markers Bmag0125 and GBMS244 (Supplementary Material, Table S1). Among those, 16 markers mapped between the flanking markers while the other 4 markers (one CAPS and three SSR) mapped near but outside the flanked QTL interval. The 16 markers consist of two SSR, two SCAR and 12 CAPS markers. Seven of the linked markers are synteny-based markers, developed using rice and $B$. distachyon annotated genes. The rice syntenic region on chromosome 4 was identified by BLAST with Uni19962 and GBM1062 sequences. Uni19962 is homologous to Loc_Os04g47040 in rice, and GBM1062 is homologous to Loc_Os04g46820 in rice. However, there is no B. distachyon homologue for Uni19962 and GBM1062. Therefore the $B$. distachyon syntenic region on chromosome Bd5 was based on the rice homologue of Uni19962 and GBM1062, as well as two EST-based markers, WBE144 and WBE129, which were flanked by Uni19962 and GBM1062. WBE144 is homologous to Bradi5g17980 in B. distachyon, and WBE129 is homologous to Bradi5g18000 in B. distachyon.

For rphq16, 27 markers were developed that supposedly mapped on chromosome 5HL in the QTL confidence interval flanked by the markers ABG391 and GMS002 (Supplementary Material, Table S2). Among those, 18 
Table 1 Summary of partial resistance QTLs against barley leaf rust isolate 1.2.1 detected at adult plant stage in S/M DH mapping population

\begin{tabular}{lllllll}
\hline QTL & Chrom & Peak marker & $\mathrm{cM}^{\mathrm{a}}$ & LOD & Exp\% & Donor \\
\hline Rphq22 & $1 \mathrm{H}$ & Contig8593 & 134.4 & 8.6 & 26.1 & Steptoe \\
Rphq23 & $3 \mathrm{H}$ & Contig10370 & 101.9 & 6.1 & 21.7 & Steptoe \\
\hline
\end{tabular}

The QTL features are based on the series with the highest LOD score using MAPQTL®6 (van Ooijen 2009)

a Peak marker position on the integrated map "Barley, Integrated, Marcel 2009"

${ }^{\mathrm{b}}$ Percentage of explained phenotypic variance (MapQTL®6)

markers mapped between the flanking markers of the QTL while the other 9 markers (1 SCAR, 5 CAPS and 3 SSR) mapped near but outside the flanked QTL interval. Three of the markers closely linked to $r p h q 16$ are syntenybased markers. The rice syntenic region on chromosome 3 and $B$. distachyon syntenic region on chromosome $\mathrm{Bd} 1$ was identified by BLAST with WBE320 and GBS0408 sequences. WBE320 is homologous to Loc_Os03g63450 in rice and Bradilg01500 in B. distachyon. GBS0408 is homologous to Loc_Os03g63940 in rice and Bradilg00990 in B. distachyon.

High-resolution genetic map for Rphq11 and rphq16

There were 89 fixed QTL recombinants identified for Rphq11 and 135 for rphq16 (described in the next section). These fixed QTL recombinants were genotyped with the newly developed markers, and highresolution genetic maps were generated (Figs. 1 and 2).

On the new high-resolution genetic map of the Rphq11 region, the distance between markers GBS0512 and GBMS244, flanking Rphq11, is approximately $6 \mathrm{cM}$. Their interval on the barley integrated map [Barley, Integrated, Marcel 2009; (Aghnoum et al. 2010)] is comparable in size (5 cM). Sixteen markers were mapped in this interval, providing an average marker density of one marker per $0.4 \mathrm{cM}$. Marker positions of GBS0512, WBE301 and GBM1062 around Rphq11 in the new-high resolution genetic map were slightly different compared to the integrated map. GBS0512 (Stein et al. 2007) and WBE301 (Potokina et al. 2008) were originally mapped in $\mathrm{S} / \mathrm{M}$, and GBM1062 in OWB (Varshney et al. 2007), which can explain the inaccuracy of their order on the integrated map. The 6-cM genetic window containing Rphq11 is in synteny with rice chromosome 4 and $B$. distachyon chromosome Bd5. The orientation of the syntenic block delimited by Uni19962 and WBE307 in barley is inverted compared to rice and B. distachyon, and microsyntenic rearrangements in marker order are also observed within the block. The orientation of the syntenic block and the order of markers are perfectly conserved between rice and B. distachyon (Fig. 1).

On the new high-resolution genetic map of the rphq16 region, the distance between markers scsnp03275 and GMS002, flanking rphq16, is approximately $11 \mathrm{cM}$. Their interval is approximately $20 \mathrm{cM}$ on the barley integrated map [Barley, Integrated, Marcel 2009; (Aghnoum et al. 2010)]. The estimated 20-cM interval was based on MWG2193, WBE310 and WBE314 which shared the same position as scsnp03275 on the high-resolution genetic map because scsnp03275 was not mapped in the integrated map.

There were 18 markers mapped in this interval, providing an average marker density of one marker per $0.6 \mathrm{cM}$. Marker order at rphq16 was in agreement with marker order on the integrated map. The 11-cM genetic window comprising rphq16 is in synteny with rice chromosome 3 and $B$. distachyon chromosome Bd1. The orientation of this syntenic region and marker order are perfectly conserved between barley and rice but inverted in B. distachyon (Fig. 2).

Fine mapping of Rphq11 and rphq16

The disease test on $97 \mathrm{~F}_{3}$ plants segregating for Rphq11 showed that Rphq11 is an incompletely dominant gene, while the disease test on $52 \mathrm{BC}_{1} \mathrm{~S}_{1}$ plants segregating for rphq16 shows that rphq16 behaves predominantly as a recessive gene (Supplementary Material, Fig. S5). The RLP50S comparison between the group of plants with homozygous donor allele (AA) and the group of plants without the donor allele (BB) shows that Rphq11 gives an approximately 21 -h (11\%) prolongation of latency period on the seedling leaves and rphq16 an approximately 14-h (7\%) prolongation. 


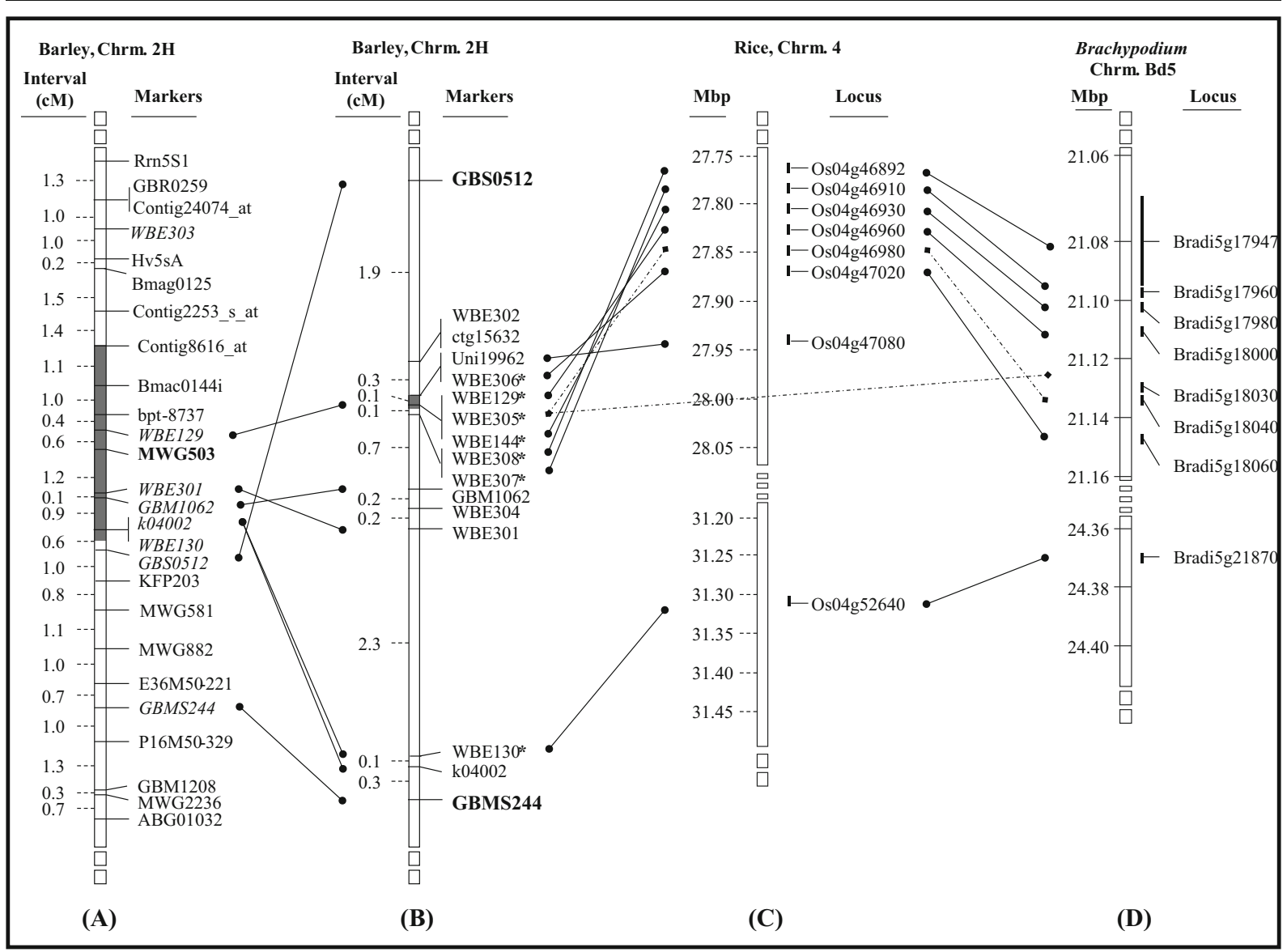

Fig. 1 Alignment of a the integrated map "Marcel 2009" and $\mathbf{b}$ the high-resolution map generated in this study, at the Rphq11 region on barley chromosome 2HL with c the physical map of rice chromosome 4 and $\mathbf{d}$ the physical map of $B$. distachyon chromosome $\mathrm{Bd} 5$. The filled grey areas inside chromosome bars indicate the position of Rphq11.

Recombinant plant screening of 730 plants at $F_{4}$ and $\mathrm{F}_{5}$ resulted in 89 fixed QTL recombinants at Rphq11 representing 10 recombination intervals between all the markers mapped between GBS0512 and GBMS244. For rphq16, recombinant plant screening of 655 plants at $\mathrm{BC}_{2} \mathrm{~S}_{1}$ and $\mathrm{BC}_{2} \mathrm{~S}_{2}$ resulted in 135 fixed QTL recombinants representing 9 recombination intervals between all the markers mapped between WBE318 and scsnp03683.

After several rounds of disease tests on a subset of the fixed QTL recombinants, Rphq11 was finemapped into a genetic window of $0.2 \mathrm{cM}$ flanked by markers Uni19962 and WBE306 proximally and WBE307 and WBE308 distally. Indeed, the peak of the LOD profile generated by performing QTL
The bold marker on a is the peak marker of Rphq11. The bold markers on $\mathbf{b}$ are the flanking markers used for recombinant screening, and the markers with an asterisk on $\mathbf{b}$ are synteny-based markers. The dashed lines show homologous sequences found between only two of the three species barley, rice and $B$. distachyon

mapping on the fixed QTL recombinants supports this position of Rphq11 (Fig. 3a). This is consistent with an RLP50S between 107 and 110 for fixed QTL recombinants having the Rphq11 allele, which is always significantly higher than the RLP50S on SusPtrit. And this is consistent with an RLP50S between 101 and 107 for fixed QTL recombinants having the rphq11 allele, which is however not always significantly shorter than those having the Rphq11 allele and, in some cases, significantly longer than on the susceptible SusPtrit.

Similarly, rphq16 was fine-mapped into a genetic window of $1.4 \mathrm{cM}$ flanked by markers WBE313 proximally and MWG2249 and WBE320 distally. The peak of the LOD profile generated by 


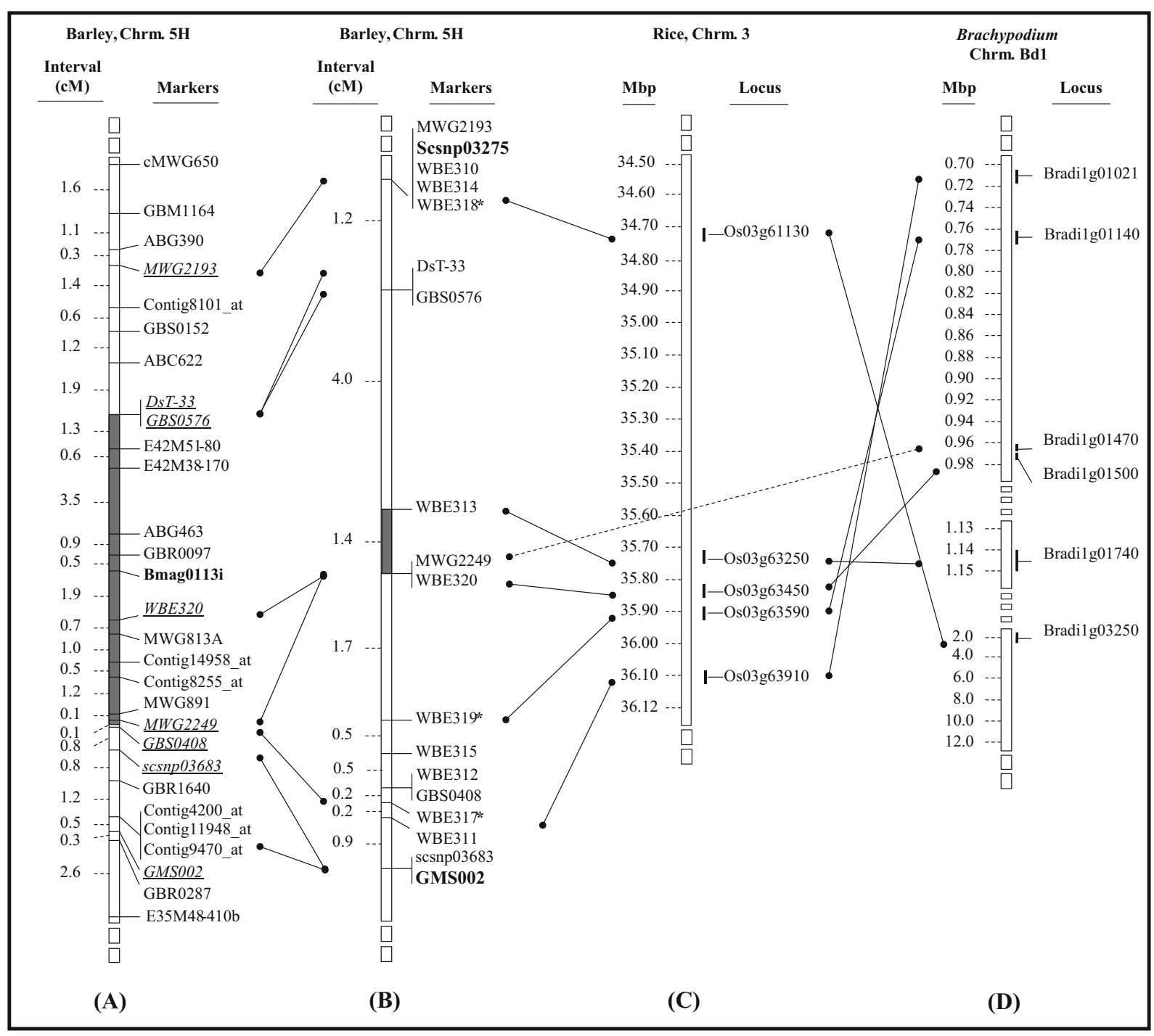

Fig. 2 Alignment of a the integrated map "Marcel 2009" and $\mathbf{b}$ the high-resolution map generated in this study, at the rphq16 region on barley chromosome 5HL with $\mathbf{c}$ the physical map of rice chromosome 3 and $\mathbf{d}$ the physical map of $B$. distachyon $\mathrm{Bd} 1$. The filled grey areas inside chromosome bars indicate the position of rphq16. The bold marker on $\mathbf{a}$ is the peak marker of rphq16. The bold markers on $\mathbf{b}$ are the flanking markers used for recombinant screening, and the markers with an asterisk on $\mathbf{b}$ are synteny-based markers. The dashed line shows a homologous sequence between barley and $B$. distachyon, which was only found in rice on another chromosome performing QTL mapping on the fixed QTL recombinants supports this position of rphq16 (Fig. 3b). This is consistent with RLP50S on fixed QTL recombinants having the rphq16 allele ranging from 106 to 111 , which is always significantly longer than the RLP50S on SusPtrit. And this is also consistent with RLP50S on fixed QTL recombinants having the Rphq16 allele ranging from 100 to 105 , which is not always significantly shorter than those having the rphq16 allele and, in some cases, significantly longer than on the susceptible SusPtrit.

The refined position of Rphq11 in a 0.2 -cM interval corresponds to the syntenic region inverted between barley and rice (Fig. 1). The $0.2 \mathrm{cM}$ in barley corresponds to physical distances of $161 \mathrm{~kb}$ with 18 annotated genes in rice and $79 \mathrm{~kb}$ with 9 annotated genes in B. distachyon. Concerning rphq16, its refined position of $1.4 \mathrm{cM}$ in barley corresponds to physical distances of 


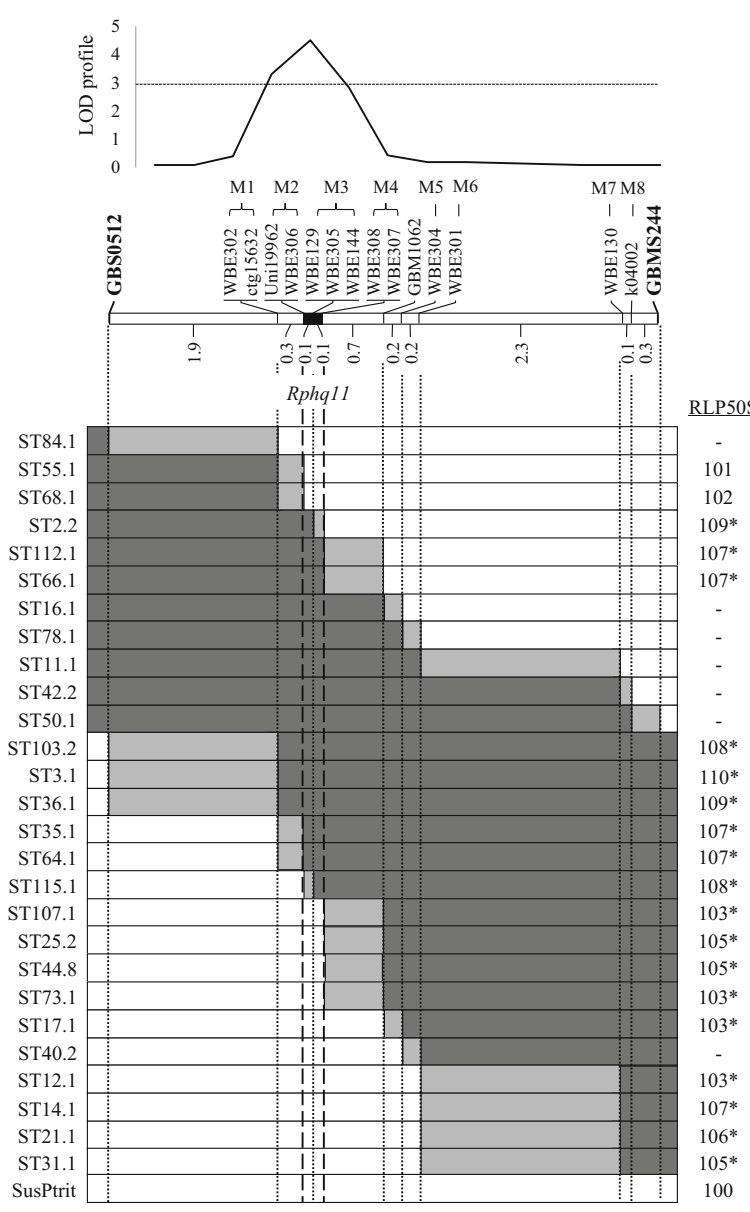

(A)

Fig. 3 Graphical genotypes and phenotype means (RLP50S) for fixed QTL recombinants of a Rphq11 and b rphq16; the phenotype means were compiled from results of the different rounds of disease test. The white bars represent homozygous SusPtrit. Black bars represent homozygous Steptoe (a) or Dom (b). Grey bars represent intervals where

$118 \mathrm{~kb}$ with 20 annotated genes in rice and $188 \mathrm{~kb}$ with 9 annotated genes in B. distachyon.

\section{Discussion}

Plant stage-specific QTLs in S/M and OWB

The seedlings and adult plants of S/M and OWB mapping populations were challenged with $P h \cdot 1.2 .1$. None of the partial resistance QTLs that were detected at seedling stage (Marcel et al. 2007b) were also detected in any of the three repetitions of the disease test at adult plant developmental stage (this study). This indicates

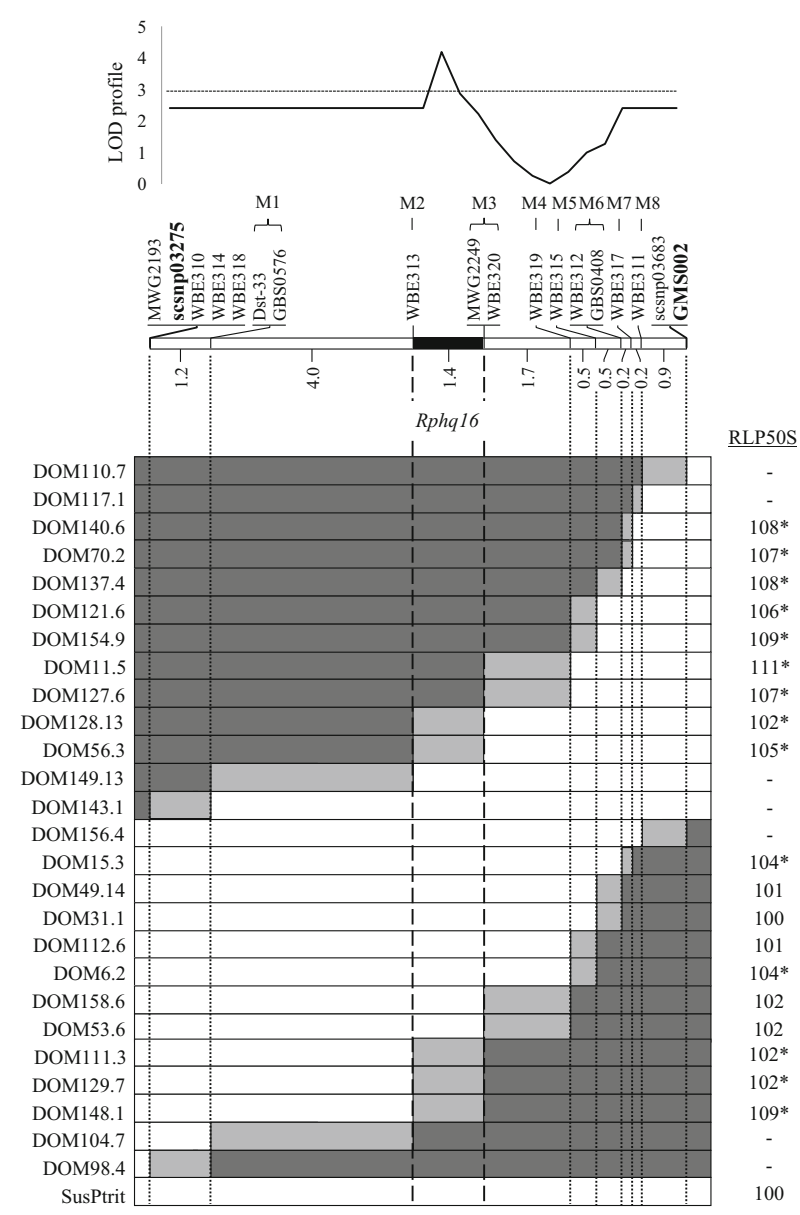

(B)

recombination took place. RLP50S values with an asterisk are significantly longer than the RLP50S on SusPtrit. The number between two markers on the chromosome bar indicates the recombination frequency observed. The new genetic window of Rphq11 and rphq16 is indicated between the long dashed lines

that Rphq11 and rphq16 are plant growth stage dependent and that their characterisation can only be performed at seedling stage. Rphq22 and Rphq23, which were detected in this study at adult stage in S/M, are also plant growth stage dependent, since they were not detected in the earlier seedling tests. This plant stage dependence of QTLs for partial resistance against $P$. hordei was also reported for other partial resistance QTLs in this pathosystem (Qi et al. 1998; Wang et al. 2010).

Plant growth characteristics, such as heading date and plant height, may influence the resistance of plants (Klahr et al. 2007; Williams 2003). In rice, the germinlike protein 1 was demonstrated to be involved in 
regulating plant height and disease resistance (Banerjee and Maiti 2010). Rphq22 and Rphq23 did not collocate with any heading date and plant height QTLs, which suggest that the resistance conferred by these two QTLs is not a pleiotropic effect of genes affecting heading date or plant height.

Adult plant resistance QTLs from S/M and OWB are affected by the environment

In both populations, a clear repetition $\times$ genotype effect was observed, which was especially strong in the OWB population. From the three QTLs mapped in S/M, one was identified in only one of the three repetitions. And all three QTLs mapped in OWB were identified in only one of the three repetitions. Environmental effects may be among the contributing factors for the inconsistency of QTL identification. The disease tests were carried out at different time periods of the year, and the third repetition was carried out at a different greenhouse facility.

The high morphological variation between the OWB lines may also have contributed to the inconsistency of QTLs mapped in this population. The parental lines of the OWB population have been developed by systematically crossing recessive alleles for morphological and physiological traits into one parent and dominant alleles into the other parent, maximizing the genetic, morphological and agronomic diversity segregating in the population (Costa et al. 2001). The variation in plant height may affect the uniformity of inoculum deposition. Heading date variation can also influence the result of a disease test as the sequential inoculation of different groups of lines according to their heading date may compromise uniformity and randomisation of $\mathrm{DH}$ lines over batches. Notably, the disease resistance of DH lines may vary based on the age of the flag leaf used. It is difficult to homogenise the disease tests for this population due to the high morphological variation between lines.

\section{Efficient fine mapping of Rphq11 and rphq16}

A QTL can be fine-mapped without the interference of other QTLs if NILs were used as starting material. The NIL development is, however, laborious and time consuming. For barley, one generation is approximately 4 months; thus, the development of a NIL with approximately $95 \%$ of its genome coming from the recurrent parent will take 2 years and 4 months to reach generation $\mathrm{BC}_{4}$. Another year will be necessary to obtain enough seeds of homozygous recombinant plants (i.e. sub-NILs) to allow fine mapping. In total, about 3 years and 4 months are needed to fine-map a QTL to a certain genetic window. One way to shorten this procedure is by exploiting individual lines from recombinant inbred lines (RILs), DH lines, chromosome segment substitution lines (CSSLs) and backcross inbred lines (BILs) (Gao et al. 2004; Liu and Bai 2010; Zhang et al. 2011). Effectively, individual line(s) which contain only the resistance allele of the targeted QTL can be crossed with a susceptible parent in order to generate recombinant plants that will be used for fine mapping. However, the construction of RILs, CSSL and BILs is as laborious and time consuming as NIL and sub-NIL development.

Tuinstra et al. (1997) proposed a method to identify QTL-NILs from populations of advanced generation inbreds, usually $\mathrm{F}_{5}$ or $\mathrm{F}_{6}$ generation inbred lines. Individuals from one inbred family segregating for a selected marker linked to the QTL of interest will be nearly isogenic allowing to confirm the phenotypic effect of the QTL and to screen for recombinants at this QTL. The fine mapping strategy followed in our study aimed at identifying recombinants in early plant material developed to produce QTL-NILs. The material was first selected to carry the targeted QTL in heterozygous condition and susceptibility alleles at other resistance QTLs in homozygous condition. This strategy took four to five generations to obtain fixed QTL recombinants. This way, it became possible to fine-map a QTL in less than 2 years. In parallel, the NILs of the targeted QTLs were developed, which allowed the confirmation of the effect of Rphq11 and rphq16 in an isogenic background (data not shown).

The position of Rphq11 has been narrowed down to a genetic window of $0.2 \mathrm{cM}$ (1460 gametes scored) and rphq16 to a genetic window of $1.4 \mathrm{cM}$ (1310 gametes scored). The fixed QTL recombinants were not monitored for the presence of donor genome outside the regions of the targeted QTLs. Theoretically, the plant materials (i.e. $\mathrm{F}_{3}$ ) used to fine-map Rphq11 should have approximately $50 \%$ of donor genome. For $r p h q 16$, the plant materials (i.e. $\mathrm{BC}_{2} \mathrm{~S}_{1}$ ) may still have approximately $13 \%$ of donor genome. Consequently, previously undetected minor effect QTLs for resistance may still be present and even segregate in the material used to fine-map Rphq11 and rphq16. As a result of this heterogeneous genetic background, we may expect genetic and hence phenotypic noise in determining the QTL position and assessment of its effect. This may explain 
that several of the fixed QTL recombinants carrying the susceptibility allele at the target QTL give a significantly longer latency period than the susceptible line SusPtrit used for crossing.

Nevertheless, it was still possible to dissect the position of Rphq11 and rphq16 as the RLP50 range between fixed QTL recombinants carrying the susceptibility allele and fixed QTL recombinants carrying the resistance allele at the corresponding QTL was nearly distinct (i.e. RLP50S was 101-107 versus 107-110 for Rphq11, and 100-105 versus 106-111 for rphq16). Moreover, the positions of Rphq11 and rphq16 were supported by mapping QTLs on the high-resolution map. The peak marker of Rphq11 became WBE129. The peak marker for rphq16 became MWG2249/WBE320, which is one of the markers flanking the window. Note that MWG2249/WBE320 has the second highest LOD score. The peak LOD score for rphq16 was in a marker interval. The new small genetic window of Rphq11 and rphq16 remains consistent with the position of their peak marker previously identified by Marcel et al. (2007b) (Figs. 1 and 2). The position of Rphq11 is in agreement with the eQTL mapping performed in the S/M population by Chen et al. (2010). The authors mapped eQTLs with measures of transcript abundance obtained in the S/M population $18 \mathrm{~h}$ after inoculation with $P$. hordei isolate 1.2.1. They analysed the correlation between the identified eQTL and the pQTL including Rphq11. They identified 54 eQTLs located in the confidence interval of Rphq11, and 6 genes were proposed as candidate genes for Rphq11. Of these six, 'Unigene2453' encoding a phospholipid hydroperoxide glutathione peroxidase (PHGPx) was considered the strongest candidate for Rphq11 (Chen et al. 2010). Interestingly, the marker developed on Unigene2453, WBE129, indeed was the peak marker of Rphq11 located within the refined 0.2-cM position of this PQTL. The six most strong candidates listed by Chen et al. (2010) all have homologues in rice, but out of those six, only the gene corresponding to PHGPx (Os04g46960) is located in the syntenic region corresponding with the barley WBE306 to WBE307 interval.

Rphq11 and rphq16 are the second and third finemapped QTLs for barley partial resistance to leaf rust. The fine mapping strategy followed in this study has proven efficient to fine-map these two QTLs with a slightly smaller effect on the resistance level than $R p h q 2$. The latter gene explained $50 \%$ of the phenotypic variance and was fine-mapped in another study (Marcel et al. 2007a). However, in order to fine-map smallereffect QTLs, it would probably be necessary to reduce the noise caused by genetic background by starting the fine mapping process at $\mathrm{BC}_{3}$ or at even later backcross generations as suggested by Yang et al. (2012).

Identification of candidate genes for Rphq11 and $r p h q 16$ based on synteny between barley, rice and $B$. distachyon

The genetic window of Rphq11 is syntenic with rice chromosome 4, in agreement with Pourkheirandish et al. (2007), and with B. distachyon chromosome Bd5 (Mayer et al. 2011). The orientation of the syntenic region corresponding to Rphql1 is conserved between rice and $B$. distachyon but inverted in barley. The size of the Rphq11 syntenic region in rice is approximately $161 \mathrm{~kb}$, with 18 annotated genes. In $B$. distachyon, the size is approximately $79 \mathrm{~kb}$ with nine annotated genes. None of the genes found in this region possess a nucleotide-binding site (NBS) or a leucine-rich repeat (LRR) domain commonly found in major resistance genes (DeYoung and Innes 2006). However, several genes belong to gene families previously shown to be involved in resistance in other plant pathogen systems. These genes include an actin-depolymerizing factor (Wang et al. 2013), a glutathione peroxidase (Lamb and Dixon 1997) and glycosyltransferases (LangloisMeurinne et al. 2005; von Saint et al. 2011). Homologues of all those genes are found in the syntenic region of both rice and $B$. distachyon.

Glutathione peroxidase is the most promising candidate gene for Rphq11. The peak marker for Rphq11 on the high-resolution genetic map is WBE129, which has been developed on the candidate gene Unigene2453 encoding for the phospholipid hydroperoxide glutathione peroxidase (PHGPx). This PHGPx gene has also been identified as the strongest candidate to explain Rphq11 by Chen et al. (2010), because it was detected as a high-LOD cis-regulated expressed QTL with significantly different transcript abundances between Steptoe and Morex. Out of the six strongest candidate genes emerging from the work of Chen et al. (2010), it is the only gene located in the syntenic rice interval (see above). As this PHGPx gene is conserved across rice, $B$. distachyon and barley, it may also have a conserved function in defence against pathogens across plant species. In rice, the expression of rice PHGPx homologue - OsPHGPx - is induced by infection with 
Magnaporthe grisea (Agrawal et al. 2002). The tomato PHGPx homologue - LePHGPx - also confers resistance, against Botrytis cinerea, when stably expressed in tobacco (Chen et al. 2004).

The genetic window of rphq16 is syntenic with rice chromosome 3, in agreement with Close et al. (2009), and B. distachyon chromosome Bd1 (Mayer et al. 2011). The orientation of the syntenic region corresponding to rphq16 is conserved between barley and rice, but it is inverted in B. distachyon. The size of the syntenic region is approximately $118 \mathrm{~kb}$ with 20 annotated genes in rice and $188 \mathrm{~kb}$ with 9 annotated genes in B. distachyon. Several of these genes belong to gene families involved in resistance in other plant pathogen systems, including an oxidoreductase (Montesano et al. 2003), an aspartokinase (Stuttmann et al. 2011) and a proteasome subunit (Yao et al. 2012), which are conserved between rice and $B$. distachyon. There is also a glutathione Stransferase (Dean et al. 2005) and a transcription factor BTF3 (Huh et al. 2012) found only in the rice syntenic region, as well as a protein kinase C (Subramaniam et al. 1997), protein tyrosine phosphatases (He et al. 2012), glycosyltransferases (Langlois-Meurinne et al. 2005) and a NBS-LRR gene (Loutre et al. 2009) found only in the $B$. distachyon syntenic region. There is no favourite candidate gene in the interval for the moment.

\section{Feasibility of map-based cloning Rphq11 and rphq16}

Rphq11 or rphq16 prolongs the latency period of $P$. hordei isolate 1.2.1. by $12 \mathrm{~h}$ in comparison to SusPtrit, which proved to be sufficient to differentiate plants with the resistance allele from those with the susceptibility allele at each QTL. In agreement with this observation, the effect of Rphq11 and of rphq16 was confirmed in isogenic lines (data not shown). It allowed fine mapping Rphq11 and rphq16 to barley regions of 0.2 and $1.4 \mathrm{cM}$, respectively, following a time-efficient strategy. Even though there is phenotypic noise, it was still possible to dissect the position of Rphq11 and rphq16. Rphq11 was mapped in a high recombination rate region $(1.1 \mathrm{Mb} / \mathrm{cM})$ of approximately $220 \mathrm{~kb}$ from barley chromosome $2 \mathrm{H}$ and $r p h q 16$ in a very high recombination rate region $(0.2-0.9 \mathrm{Mb} / \mathrm{cM})$ of approximately 200 $900 \mathrm{~kb}$ from barley chromosome $5 \mathrm{H}$ (Künzel et al. 2000). This offers good prospects for the map-based cloning of the gene(s) underlying these two QTLs.

Many QTLs for partial resistance were mapped in barley against barley leaf rust, but the underlying genes have not been identified so far. $R p h q 2$ has previously been fine-mapped to a genetic interval of $0.11 \mathrm{cM}$ (Marcel et al. 2007a), encompassing a barley region of approximately $190 \mathrm{~kb}$. This area comprises about 12 genes in partially resistant Vada, of which 7 do not correspond to any gene in the susceptible parent SusPtrit (Yeo et al. 2015). The precise mapping of Rphq11 and rphq16 is a new step towards the understanding of the genetic basis of partial resistance to barley leaf rust. The sequenced Morex genome (The International Barley Genome Sequencing Consortium 2012) can be the reference for constructing the physical maps of Steptoe and Dom and for identifying candidate genes for Rphq11 and rphq16. If the genes for $R p h q 11$ and $r p h q 16$ are not present in Morex, bacterial artificial chromosomes (BACs) for Steptoe and Dom should be constructed in order to build physical maps and identify candidate genes. The identification of the gene(s) for Rphq2, Rphq11 and rphq16 will reveal if the genes for barley partial resistance belong to the same or different gene families.

Acknowledgements The authors thank their colleagues in IPK at Gatersleben, Germany, for permission to use the confidential sequences to run three SSR markers listed in Tables S1 and S3. The authors wish to thank the University Malaysia Sarawak, Malaysia, for granting the fellowship to FKS Yeo to work on this $\mathrm{PhD}$ thesis in Wageningen and Prof RGF Visser for hosting FKS Yeo for that purpose at the Laboratory of Plant Breeding. We particularly wish to thank Anton Vels for his careful maintenance of plant materials.

\section{Compliance with ethical standards}

Conflict of interest The authors declare that they have no conflict of interest.

Open Access This article is distributed under the terms of the Creative Commons Attribution 4.0 International License (http:// creativecommons.org/licenses/by/4.0/), which permits unrestricted use, distribution, and reproduction in any medium, provided you give appropriate credit to the original author(s) and the source, provide a link to the Creative Commons license, and indicate if changes were made.

\section{References}

Aghnoum R, Marcel TC, Johrde A, Pecchioni N, Schweizer P, Niks RE (2010) Basal host resistance of barley to powdery mildew: connecting quantitative trait loci and candidate genes. Mol Plant-Microbe Interact 23:91-102

Agrawal GK, Rakwal R, Jwa N, Agrawal VP (2002) Effects of signaling molecules, protein phosphatase inhibitors and blast pathogen (Magnaporthe grisea) on the mRNA level of a rice 
(Oryza sativa L.) phospholipid hydroperoxide glutathione peroxidase (OsPHGPX) gene in seedling leaves. Gene 283: 227-236

Atienza S, Jafary H, Niks RE (2004) Accumulation of genes for susceptibility to rust fungi for which barley is nearly a nonhost results in two barley lines with extreme multiple susceptibility. Planta 220:71-79

Banerjee J, Maiti MK (2010) Functional role of rice germin-like protein1 in regulation of plant height and disease resistance. Biochem and Bioph Res Co 394:178-183

Boyd CN, Horsley R, Kleinhofs A (2007) Barley chromosome 2 $(2 \mathrm{H})$ bin 10 fusarium head blight resistance QTL: mapping and development of isolines. In: Canty SM, Clark A, Ellis D, van Sanford D (eds) National Fusarium Head Blight Forum 2007. Michigan State University, Kansas City, MO, East Lansing, pp 170-172

Catanzariti A, Dodds PN, Ellis JG (2007) Avirulence proteins from haustoria-forming pathogens. FEMS Microbiol Lett 269:181-188

Chen S, Vaghchhipawala Z, Li W, Asard H, Dickman MB (2004) Tomato phospholipid hydroperoxide glutathione peroxidase inhibits cell death induced by bax and oxidative stresses in yeast and plants. Plant Physiol 135:1630-1641

Chen X, Hackett CA, Niks RE, Hedley PE, Booth C, Druka A, Marcel TC, Vels A, Bayer M, Milne I (2010) An eQTL analysis of partial resistance to Puccinia hordei in barley. PLoS One 5(1):e8598

Close T, Bhat P, Lonardi S, Wu Y, Rostoks N, Ramsay L, Druka A, Stein N, Svensson J, Wanamaker S, Bozdag S, Roose M, Moscou M, Chao S, Varshney R, Szucs P, Sato K, Hayes P, Matthews D, Kleinhofs A, Muehlbauer G, DeYoung J, Marshall D, Madishetty K, Fenton R, Condamine P, Graner A, Waugh R (2009) Development and implementation of high-throughput SNP genotyping in barley. BMC Genomics 10:582

Costa JM, Corey A, Hayes PM, Jobet C, Kleinhofs A, KopischObusch A, Kramer SF, Kudrna D, Li M, Riera-Lizarazu O (2001) Molecular mapping of the Oregon Wolfe Barleys: a phenotypically polymorphic doubled-haploid population. Theor Appl Genet 103:415-424

de Jonge R, Bolton MD, Thomma BPHJ (2011) How filamentous pathogens co-opt plants: the ins and outs of fungal effectors. Curr Opin Plant Biol 14:400-406

Dean JD, Goodwin PH, Hsiang T (2005) Induction of glutathione S-transferase genes of Nicotiana benthamiana following infection by Colletotrichum destructivum and C. orbiculare and involvement of one in resistance. J Exp Bot 56:1525-1533

Dedryver F, Paillard S, Mallard S, Robert O, Trottet M, Nègre S, Verplancke G, Jahier J (2009) Characterization of genetic components involved in durable resistance to stripe rust in the bread wheat 'Renan'. Phytopathology 99:968-973

DeYoung BJ, Innes RW (2006) Plant NBS-LRR proteins in pathogen sensing and host defense. Nat Immunol 7:1243-1249

Fu D, Uauy C, Distelfeld A, Blechl A, Epstein L, Chen X, Sela H, Fahima T, Dubcovsky J (2009) A kinase-START gene confers temperature-dependent resistance to wheat stripe rust. Science 323:1357-1360

Fukuoka S, Saka N, Koga H, Ono K, Shimizu T, Ebana K, Hayashi N, Takahashi A, Hirochika H, Okuno K, Yano M (2009) Loss of function of a proline-containing protein confers durable disease resistance in rice. Science 325:998-1001
Gao W, Clancy JA, Han F, Jones BL, Budde A, Wesenberg DM, Kleinhofs A, Ullrich SE (2004) Fine mapping of a maltingquality QTL complex near the chromosome 4HS telomere in barley. Theor Appl Genet 109:750-760

González AM, Marcel TC, Niks RE (2012) Evidence for a minor gene-for-minor gene interaction explaining nonhypersensitive polygenic partial disease resistance. Phytopathology 102:1086-1093

Han F, Ullrich SE, Clancy JA, Romagosa I (1999) Inheritance and fine mapping of a major barley seed dormancy QTL. Plant Sci 143:113-118

Hayashi N, Inoue H, Kato T, Funao T, Shirota M, Shimizu T, Kanamori H, Yamane H, Hayano-Saito Y, Matsumoto T, Yano M, Takatsuji H (2010) Durable panicle blastresistance gene $\mathrm{Pbl}$ encodes an atypical CC-NBS-LRR protein and was generated by acquiring a promoter through local genome duplication. Plant J 64:498-510

Hayes PM, Liu BH, Knapp SJ, Chen F, Jones B, Blake T, Franckowiak J, Rasmusson D, Sorrells M, Ullrich SE, Wesenberg D, Kleinhofs A (1993) Quantitative trait locus effects and environmental interacion in a sample of North American barley germ plasm. Theor Appl Genet 87:392-401

He H, Su J, Shu S, Zhang Y, Ao Y, Liu B, Feng D, Wang J, Wang $\mathrm{H}$ (2012) Two homologous putative protein tyrosine phosphatases, OsPFA-DSP2 and AtPFA-DSP4, negatively regulate the pathogen response in transgenic plants. PLoS One 7(4):e34995

Huh SU, Kim K, Paek K (2012) Capsicum annuum basic transcription factor $3(\mathrm{CaBtf} 3)$ regulates transcription of pathogenesis-related genes during hypersensitive response upon tobacco mosaic virus infection. Biochem and Bioph Res Co 417:910-917

Jafary H, Albertazzi G, Marcel TC, Niks RE (2008) High diversity of genes for nonhost resistance of barley to heterologous rust fungi. Genetics 178:2327-2339

Klahr A, Zimmermann G, Wenzel G, Mohler V (2007) Effects of environment, disease progress, plant height and heading date on the detection of QTLs for resistance to Fusarium head blight in an European winter wheat cross. Euphytica 154:17-28

Kleinhofs A, Kilian A, Saghai Maroof MA, Biyashev RM, Hayes P, Chen FQ, Lapitan N, Fenwick A, Blake TK, Kanazin V (1993) A molecular, isozyme and morphological map of the barley (Hordeum vulgare) genome. Theor Appl Genet 86: 705-712

Kou Y, Wang S (2012) Toward an understanding of the molecular basis of quantitative disease resistance in rice. J Biotechnol 159:283-290

Krattinger SG, Lagudah ES, Spielmeyer W, Singh RP, Huerta-Espino J, McFadden H, Bossolini E, Selter LL, Keller B (2009) A putative $\mathrm{ABC}$ transporter confers durable resistance to multiple fungal pathogens in wheat. Science 323:1360-1363

Künzel G, Korzun L, Meister A (2000) Cytologically integrated physical restriction fragment length polymorphism maps for the barley genome based on translocation breakpoints. Genetics 154:397-412

Lamb C, Dixon RA (1997) The oxidative burst in plant disease resistance. Annu Rev Plant Physiol Plant Molec Biol 48: 251-275

Langlois-Meurinne M, Gachon CMM, Saindrenan P (2005) Pathogen-responsive expression of glycosyltransferase genes UGT73B3 and UGT73B5 is necessary for resistance to 
Pseudomonas syringae pv tomato in Arabidopsis. Plant Physiol 139:1890-1901

Liu S, Bai G (2010) Dissection and fine mapping of a major QTL for preharvest sprouting resistance in white wheat Rio Blanco. Theor Appl Genet 121:1395-1404

Loutre C, Wicker T, Travella S, Galli P, Scofield S, Fahima T, Feuillet C, Keller B (2009) Two different CC-NBS-LRR genes are required for $\mathrm{Lr10}$-mediated leaf rust resistance in tetraploid and hexaploid wheat. Plant J 60:1043-1054

Manosalva PM, Davidson RM, Liu B, Zhu X, Hulbert SH, Leung H, Leach JE (2009) A germin-like protein gene family functions as a complex quantitative trait locus conferring broad-spectrum disease resistance in rice. Plant Physiol 149:286-296

Marcel TC, Aghnoum R, Durand J, Varshney RK, Niks RE (2007a) Dissection of the barley 2 L1. 0 region carrying the 'Laevigatu' quantitative resistance gene to leaf rust using near-isogenic lines (NIL) and subNIL. Mol Plant-Microbe Interact 20:1604-1615

Marcel TC, Varshney RK, Barbieri M, Jafary H, de Kock MJD, Graner A, Niks RE (2007b) A high-density consensus map of barley to compare the distribution of QTLs for partial resistance to Puccinia hordei and of defence gene homologues. Theor Appl Genet 114:487-500

Marcel TC, Gorguet B, Ta MT, Kohutova Z, Vels A, Niks RE (2008) Isolate specificity of quantitative trait loci for partial resistance of barley to Puccinia hordei confirmed in mapping populations and near-isogenic lines. New Phytol 177:743-755

Mayer KFX, Martis M, Hedley PE, Šimková H, Liu H, Morris JA, Steuernagel B, Taudien S, Roessner S, Gundlach H, Kubaláková M, Suchánková $\mathrm{P}$, Murat F, Felder M, Nussbaumer T, Graner A, Salse J, Endo T, Sakai H, Tanaka T, Itoh T, Sato K, Platzer M, Matsumoto T, Scholz U, Doležel J, Waugh R, Stein N (2011) Unlocking the barley genome by chromosomal and comparative genomics. Plant Cell 23: $1249-1263$

Montesano M, Hyytiäinen H, Wettstein R, Palva ET (2003) A novel potato defence-related alcohol: $\mathrm{NADP}^{+}$oxidoreductase induced in response to Erwinia carotovora. Plant Mol Biol 52:177-189

Moore JW, Herrera-Foessel S, Lan C, Schnippenkoetter W, Ayliffe M, Huerta-Espino J, Lillemo M, Viccars L, Milne R, Periyannan S, Kong X, Spielmeyer W, Talbot M, Bariana H, Patrick JW, Dodds P, Singh R, Lagudah E (2015) A recently evolved hexose transporter variant confers resistance to multiple pathogens in wheat. Nat Genet 47:1494-1500

Neff MM, Turk E, Kalishman M (2002) Web-based primer design for single nucleotide polymorphism analysis. Trends Genet 18:613-615

Niks RE (1983) Comparative histology of partial resistance and the nonhost reaction to leaf rust pathogens in barley and wheat seedlings. Phytopathology 73:60-64

Niks RE (1986) Failure of haustorial development as a factor in slow growth and development of Puccinia hordei in partially resistant barley seedlings. Physiol Mol Plant P 28:309-322

Niks RE (1989) Induced accessibility and inaccessibility of barley cells in seedling leaves inoculated with two leaf rust species. J Phytopath 124:296-308

Niks RE, Fernandez E, van Haperen B, Bekele Aleye B, Martinez F (2000) Specificity of QTLs for partial and non-host resistance of barley to leaf rust fungi. Acta Phytopathol Entomol Hun 35:13-21
Niks RE, Parlevliet JE, Lindhout P, Bai Y (2011) Breeding crops with resistance to diseases and pests. Wageningen Academic Publishers, Wageningen, p 198pp

Niks RE, Qi X, Marcel TC (2015) Quantitative resistance to biotrophic filamentous plant pathogens: concepts, misconceptions and mechanisms. Annu Rev Phytopathol 53:445470

O'Connell RJ, Panstruga R (2006) Tête à tête inside a plant cell: establishing compatibility between plants and biotrophic fungi and oomycetes. New Phytol 171:699-718

Parlevliet JE (1976) Evaluation of the concept of horizontal resistance in the barley/Puccinia hordei host-pathogen relationship. Phytopathology 66:494-497

Parlevliet JE (1979) Components of resistance that reduce the rate of epidemic development. Annu Rev Phytopathol 17:203-222

Parlevliet JE, Zadoks JC (1977) The integrated concept of disease resistance: a new view including horizontal and vertical resistance in plants. Euphytica 26:5-21

Potokina E, Druka A, Luo Z, Wise R, Waugh R, Kearsey M (2008) Gene expression quantitative trait locus analysis of 16000 barley genes reveals a complex pattern of genome-wide transcriptional regulation. Plant J 53:90-101

Pourkheirandish M, Wicker T, Stein N, Fujimura T, Komatsuda T (2007) Analysis of the barley chromosome 2 region containing the six-rowed spike gene vrs1 reveals a breakdown of rice-barley micro collinearity by a transposition. Theor Appl Genet 114:1357-1365

Qi X, Niks RE, Stam P, Lindhout P (1998) Identification of QTLs for partial resistance to leaf rust (Puccinia hordei) in barley. Theor Appl Genet 96:1205-1215

Qi X, Jiang G, Chen W, Niks RE, Stam P, Lindhout P (1999) Isolate-specific QTLs for partial resistance to Puccinia hordei in barley. Theor Appl Genet 99:877-884

Shankar M, Walker E, Golzar H, Loughman R, Wilson RE, Francki MG (2008) Quantitative trait loci for seedling and adult plant resistance to Stagonospora nodorum in wheat. Phytopathology 98:886-893

Steffenson BJ, Hayes PM, Kleinhofs A (1996) Genetics of seedling and adult plant resistance to net blotch (Pyrenophora teresf. teres) and spot blotch (Cochliobolus sativus) in barley. Theor Appl Genet 92:552-558

Stein N, Prasad M, Scholz U, Thiel T, Zhang H, Wolf M, Kota R, Varshney RK, Perovic D, Grosse I, Graner A (2007) A 1,000loci transcript map of the barley genome: new anchoring points for integrative grass genomics. Theor Appl Genet 114:823-839

Stuttmann J, Hubberten H, Rietz S, Kaur J, Muskett P, Guerois R, Bednarek P, Hoefgen R, Parker JE (2011) Perturbation of Arabidopsis amino acid metabolism causes incompatibility with the adapted biotrophic pathogen Hyaloperonospora arabidopsidis. Plant Cell 23:2788-2803

Subramaniam R, Després C, Brisson N (1997) A functional homolog of mammalian protein kinase $C$ participates in the elicitor-induced defense response in potato. The Plant Cell Online 9:653-664

The International Barley Genome Sequencing Consortium (2012) A physical, genetic and functional sequence assembly of the barley genome. Nature 491:711-716

Tuinstra MR, Ejeta G, Goldsbrough PB (1997) Heterogeneous inbred family (HIF) analysis: a method for developing near- 
isogenic lines that differ at quantitative trait loci. Theor Appl Genet 95:1005-1011

van Ooijen JW (2009) MapQTL® 6, software for the mapping of quantitative trait loci in experimental populations of diploid species. Kyazma B V, Wageningen

Varshney R, Marcel TC, Ramsay L, Russell J, Röder MS, Stein N, Waugh R, Langridge P, Niks RE, Graner A (2007) A high density barley microsatellite consensus map with 775 SSR loci. Theor Appl Genet 114:1091-1103

von Saint PV, Zhang W, Kanawati B, Geist B, Faus-Keßler T, Schmitt-Kopplin P, Schäffner AR (2011) The Arabidopsis glucosyltransferase UGT76B1 conjugates isoleucic acid and modulates plant defense and senescence. Plant Cell 23:4124-4145

Wang H, Qi M, Cutler AJ (1993) A simple method of preparing plant samples for PCR. Nucleic Acids Res 21:4153-4154

Wang L, Wang Y, Wang Z, Marcel TC, Niks RE, Qi X (2010) The phenotypic expression of QTLs for partial resistance to barley leaf rust during plant development. Theor Appl Genet 121:857-864

Wang X, Richards J, Gross T, Druka A, Kleinhofs A, Steffenson B, Acevedo M, Brueggeman R (2013) The rpg4-mediated resistance to wheat stem rust (Puccinia graminis) in barley (Hordeum vulgare) requires Rpg5, a second NBS-LRR gene, and an actin depolymerization factor. Mol Plant-Microbe Interact 26:407-418
Williams KJ (2003) The molecular genetics of disease resistance in barley. Crop Pasture Sci 54:1065-1079

Xue S, Li G, Jia H, Xu F, Lin F, Tang M, Wang Y, An X, Xu H, Zhang L, Kong Z, Ma Z (2010) Fine mapping Fhb4, a major QTL conditioning resistance to Fusarium infection in bread wheat (Triticum aestivum L.). Theor Appl Genet 121:147-156

Yang Q, Zhang D, Xu M (2012) A sequential quantitative trait locus fine-mapping strategy using recombinant-derived progeny. J Integr Plant Biol 54:228-237

Yao C, Wu Y, Nie H, Tang D (2012) RPN1a, a 26S proteasome subunit, is required for innate immunity in Arabidopsis. Plant J 71:1015-1028

Yeo FKS, Wang Y, Vozabova T, Huneau C, Leroy P, Chalhoub B, Qi XQ, Niks RE, Marcel TC (2015) Haplotype divergence and multiple candidate genes at Rphq2, a partial resistance QTL of barley to Puccinia hordei. Theor Appl Genet 129:289-304

Zadoks JC, Chang TT, Konzak CF (1974) A decimal code for the growth stages of cereals. Weed Res 14:415-421

Zhang Y, Wang Q, Jiang L, Liu L, Wang B, Shen Y, Cheng X, Wan J (2011) Fine mapping of qSTV11 KAS, a major QTL for rice stripe disease resistance. Theor Appl Genet 122:1591-1604

Zhou L, Zeng Y, Zheng W, Tang B, Yang S, Zhang H, Li J, Li Z (2010) Fine mapping a QTL qCTB7 for cold tolerance at the booting stage on rice chromosome 7 using a near-isogenic line. Theor Appl Genet 121:895-905 